\title{
Quadratic operators used in deducing exact ground states for correlated systems: ferromagnetism at half filling provided by a dispersive band.
}

\author{
István Chalupa and Zsolt Gulácsi \\ Department of Theoretical Physics, University of Debrecen, H-4010 Debrecen, Hungary.
}

(Dated: October 23, 2018)

\begin{abstract}
Quadratic operators are used in transforming the model Hamiltonian $\hat{H}$ of one correlated and dispersive band in an unique positive semidefinite form coopting both the kinetic and interacting part of the $\hat{H}$. The expression is used in deducing exact ground states which are minimum energy eigenstates only of the full Hamiltonian. It is shown in this frame that at half filling, also dispersive bands can provide ferromagnetism in exact terms by correlation effects .
\end{abstract}




\section{INTRODUCTION}

Exact results for non-integrable many body models open new perspectives in the description and understanding of physical systems of large interest, or physical situations capturing attention by unusual properties. Metallic ferromagnetism $\stackrel{1}{ }$, superconductivity in ladder systems ${ }^{2}$, exotic phases in polymer chains ${ }^{3}$, subtle conducting and insulating phases in rare earth compounds ${ }^{4}$, spontaneous ferroelectricity ${ }^{\underline{5}}$, stripes or checkerboards in two dimensions $^{6}$, or metal-insulator transition in two dimensional disordered systems ${ }^{7}$ are some examples in this respect.

On the technical side this field is in fact almost completely open, hence several procedures have been tested or are under development. Without to be exhaustive, we exemplify below some of these, as for example process definition followed by unapproximated treatment of the model conditions ${ }^{\underline{8}}$, Hilbert space properties captured in the low density limit $\underline{9}$, equal rigorous upper and lower bounds for the ground state energy ${ }^{10}$, unitary transformation of eigenfunctions and Hamiltonians ${ }^{11}$, Schrieffer-Wolff transformations effectuated up to infinite order $\underline{12}$, the optimal ground state method $\underline{13}$, or the use of positive semidefinite operators and related techniques ${ }^{14}$. In the present paper we concentrate on, and develop the last of these methods since the procedure based on positive semidefinite operators often penetrates in other techniques as well.

By using positive semidefinite operators $(\hat{P})$ in deducing exact ground states $\left(\left|\Psi_{g}\right\rangle\right)$, one first casts the Hamiltonian $(\hat{H})$ in a positive semidefinite form $\hat{H}=\hat{P}+C$, where $C$ is a constant depending on the coupling constants of the starting $\hat{H}$. Since zero is the minimum possible eigenvalue of $\hat{P}$, the unapproximated ground state is obtained from the requirement $\hat{P}\left|\Psi_{g}\right\rangle=0$. In the process of transforming the starting Hamiltonian in a positive semidefinite form, mostly one finds or uses $\hat{P}=\sum_{n}^{n_{\max }} \hat{P}_{n}$, where the number $n_{\max }$ of different types of positive semidefinite terms often can reach even $n_{\max }=8-10$, their structure being diverse, containing linear, bi-linear, cubic or quartic combinations of the starting fermionic operators $\frac{15}{}$. The requirement for the ground state in the presence of different $\hat{P}_{n}$ contributions becomes $\hat{P}_{n}\left|\Psi_{g}\right\rangle=0$ for all $n \in\left[1, n_{\max }\right]$, hence the deduced $\left|\Psi_{g}\right\rangle$ represents separately the ground state of different parts of the Hamiltonian, often being the ground state separately of the kinetic and interacting part of $\hat{H}$, which reduces considerably the application possibilities of the method. 
In this paper we overcome this inconvenience by using quadratic, e.g. nonlinear combinations of the starting fermionic operators in defining new non-fermionic operators $\hat{A}_{\mathbf{i}}$. Here the index $\mathbf{i}$ represents a lattice site, but $\hat{A}_{\mathbf{i}}$ is not local, has a finite extension since captures contributions also from the neighborhood of the site $\mathbf{i}$. The operators $\hat{A}_{\mathbf{i}}$ are used in the construction of an unique $\hat{G}=\sum_{\mathbf{i}} \hat{A}_{\mathbf{i}}^{\dagger} \hat{A}_{\mathbf{i}}$ positive semidefinite form, leading to the expression of the transformed Hamiltonian $\hat{H}=\hat{G}+C$, containing an unique positive semidefinite operator, namely $\hat{G}$ (the contribution $C$, as before, being a constant). Excepting the test of this procedure taken at infinitely large on-site Coulomb repulsion ${ }^{16}$, for finite and nonzero value of the interaction this approach is for the first time applied here in this paper. The gain one achieves is that now an unique positive semidefinite operator concentrates both the kinetic and interaction part of $\hat{H}$. Hence the corresponding ground states are not independently eigenstates of the kinetic and interacting part, or independently eigenstates of different parts and terms from $\hat{H}$, but only eigenstates with minimal energy of the full Hamiltonian.

Relating the studied Hamiltonian, one concentrates here on one-band correlated itinerant systems holding as well spin-spin interactions. The model Hamiltonian is in this case of extended Hubbard type (denoted hereafter by $\hat{H}_{0}$ ) containing also spin-spin interactions $\hat{H}_{S}$, hence $\hat{H}=\hat{H}_{0}+\hat{H}_{S}$ holds. A such Hamiltonian structure is particularly often debated in connection to Kondo lattices $\frac{17}{}$ or $t-J$ type of models $\frac{18}{}$, but generally emerges in the study of strong correlation effects when additional interactions between nearest neighbours are taken into consideration ${ }^{19}$, or real materials ${ }^{20,21}$ are described. We further note that in the presence of strong correlations, similar models have been also used in the study of charge-ordered states, and metal-insulator transitions in non-ferromagnetic cases 22 .

The here deduced exact eigenstates characterize dispersive bands at half filling and are localized and fully saturated ferromagnetic ground states only of the full $\hat{H}$. At this point we remark that the exact ferromagnetic ground states at finite concentration of carriers deduced up to this moment are or related to a specific band structure (flat band ferromagnetism $\underline{23}$, or Lieb's degenerate middle band ferromagnetism in a bipartite lattice ${ }^{24}$ ), or appear given by the ability of the interaction to select the ground state from the existing minimum energy eigenstates of the kinetic (not necessarily one-particle ${ }^{25}$ ) part 15 . Our results show that arbitrary dispersive half filled bands can be ferromagnetic in exact terms. Since only the whole Hamiltonian provides these ground states, these not emerge given by a specific band structure, nor appear as interaction selected ground states from existent eigenstates of other 
parts of the Hamiltonian, but are created by both kinetic and interaction contributions, hence their eigenstate nature disappears when the interactions are turned off, or when the kinetic part is neglected (e.g. in the localized limit).

The remaining part of the paper is structured as follows. Section II. describes the model used, Section III. presents the exact transformation of the Hamiltonian in positive semidefinite form, Section IV. gives the general expression of the deduced ground states and presents the proof of the ground state nature, Section V. describes particular cases when the deduced ground states hold and analyzes the physical properties of the solutions. Finally, Section VI. concluding the paper, closes the presentation.

\section{THE USED HAMILTONIAN}

Our starting Hamiltonian $\hat{H}=\hat{H}_{0}+\hat{H}_{S}$ describes in one dimension a correlated band characterized by

$$
\begin{aligned}
\hat{H}_{0} & =\sum_{\mathbf{i}, \sigma}\left(t_{\sigma} \hat{c}_{\mathbf{i}, \sigma}^{\dagger} \hat{c}_{\mathbf{i}+1, \sigma}+H . c .\right)+U \sum_{\mathbf{i}} \hat{n}_{\mathbf{i}, \uparrow} \hat{n}_{\mathbf{i}, \downarrow}+\sum_{\mathbf{i}} \sum_{\sigma_{1}, \sigma_{2}} V_{\sigma_{1}, \sigma_{2}} \hat{n}_{\mathbf{i}, \sigma_{1}} \hat{n}_{\mathbf{i}+1, \sigma_{2}}, \\
& +\sum_{\mathbf{i}, \sigma}\left(W_{1, \sigma} \hat{n}_{\mathbf{i},-\sigma} \hat{c}_{\mathbf{i}, \sigma}^{\dagger} \hat{c}_{\mathbf{i}+1, \sigma}+W_{2, \sigma} \hat{n}_{\mathbf{i}+1,-\sigma} \hat{c}_{\mathbf{i}+1, \sigma}^{\dagger} \hat{c}_{\mathbf{i}, \sigma}+H . c .\right)
\end{aligned}
$$

where $\hat{c}_{\mathbf{i}, \sigma}^{\dagger}$ creates an electron with spin $\sigma$ at the site $\mathbf{i}, \hat{n}_{\mathbf{i}, \sigma}=\hat{c}_{\mathbf{i}, \sigma}^{\dagger} \hat{c}_{\mathbf{i}, \sigma}$ represents the particle number operator, $t_{\sigma}$ is the hopping matrix element, $U$ represents the Hubbard term, $V_{\sigma_{1}, \sigma_{2}}$ gives the density-density nearest-neighbor interaction strengths, while $W_{l, \sigma}, l=1,2$ describes the correlated hopping.

Inside the band, also a nearest neighbor spin-spin interaction is present given by

$$
\hat{H}_{S}=\sum_{\mathbf{i}}\left[J_{z} \hat{S}_{\mathbf{i}}^{z} \hat{S}_{\mathbf{i}+1}^{z}+\frac{J_{\perp}}{2}\left(\hat{S}_{\mathbf{i}}^{+} \hat{S}_{\mathbf{i}+1}^{-}+\hat{S}_{\mathbf{i}}^{-} \hat{S}_{\mathbf{i}+1}^{+}\right)\right]
$$

where $\hat{S}^{ \pm}=\hat{S}^{x} \pm i \hat{S}^{y}, \hat{\vec{S}}_{\mathbf{i}}=\sum_{\alpha, \beta} \hat{c}_{\mathbf{i}, \alpha}^{\dagger} \vec{\sigma}_{\alpha, \beta} \hat{c}_{\mathbf{i}, \beta}$, and $\vec{\sigma}_{\alpha, \beta}$ describes the Pauli matrices. During this paper periodic boundary conditions are used.

\section{THE TRANSFORMATION OF THE HAMILTONIAN}

In order to deduce exact ground states for the Hamiltonian of the problem one transforms $\hat{H}$ into an unique positive semidefinite form. In order to do this we introduce a bi-linear 
operator $\hat{A}_{\mathbf{i}}$ defined by

$$
\begin{aligned}
\hat{A}_{\mathbf{i}} & =z_{1} \hat{c}_{\mathbf{i}+1, \uparrow}^{\dagger} \hat{c}_{\mathbf{i}, \downarrow}^{\dagger}+z_{2} \hat{c}_{\mathbf{i}+1, \downarrow}^{\dagger} \hat{c}_{\mathbf{i}, \uparrow}^{\dagger}+z_{3} \hat{c}_{\mathbf{i}+1, \uparrow}^{\dagger} \hat{c}_{\mathbf{i}+1, \downarrow}^{\dagger}+z_{4} \hat{c}_{\mathbf{i}, \uparrow}^{\dagger} \hat{c}_{\mathbf{i}, \downarrow}^{\dagger} \\
& +w_{1} \hat{c}_{\mathbf{i}+1, \uparrow} \hat{c}_{\mathbf{i}, \downarrow}+w_{2} \hat{c}_{\mathbf{i}+1, \downarrow} \hat{c}_{\mathbf{i}, \uparrow}+w_{3} \hat{c}_{\mathbf{i}+1, \uparrow} \hat{c}_{\mathbf{i}+1, \downarrow}+w_{4} \hat{c}_{\mathbf{i}, \uparrow} \hat{c}_{\mathbf{i}, \downarrow}
\end{aligned}
$$

where the prefactors $z_{\theta}, w_{\theta}, \theta=1,2,3,4$ are considered site independent numerical parameters. One observes that $\sum_{\mathbf{i}} \hat{A}_{\mathbf{i}}^{\dagger} \hat{A}_{\mathbf{i}}$ reproduces the operators contained in Eqs.(1),2), and indeed one finds that the exact mapping

$$
\hat{H}=\sum_{\mathbf{i}} \hat{A}_{\mathbf{i}}^{\dagger} \hat{A}_{\mathbf{i}}-a_{1} \hat{N}-a_{0}
$$

holds, if the numerical coefficients $z_{\theta}, w_{\theta}, a_{\sigma}, a_{0}$ are defined via the following matching conditions

$$
\begin{aligned}
& \frac{a_{0}}{N_{\Lambda}}=-a_{1}=\sum_{\theta=1}^{4}\left|z_{\theta}\right|^{2}, \\
& t_{\uparrow}=z_{3}^{*} z_{2}-z_{1}^{*} z_{4}, \quad t_{\downarrow}=z_{2}^{*} z_{4}-z_{3}^{*} z_{1}, \\
& V_{\uparrow, \uparrow}+\frac{J_{z}}{4}=V_{\downarrow, \downarrow}+\frac{J_{z}}{4}=0, \\
& V_{\uparrow, \downarrow}-\frac{J_{z}}{4}=\left|z_{2}\right|^{2}+\left|w_{2}\right|^{2}, \quad V_{\downarrow, \uparrow}-\frac{J_{z}}{4}=\left|z_{1}\right|^{2}+\left|w_{1}\right|^{2}, \\
& U=\left|z_{3}\right|^{2}+\left|z_{4}\right|^{2}+\left|w_{3}\right|^{2}+\left|w_{4}\right|^{2}, \quad \frac{J_{\perp}}{2}=w_{1}^{*} w_{2}+z_{2}^{*} z_{1}, \\
& w_{3}^{*} w_{1}+z_{1}^{*} z_{3}=W_{2, \downarrow}, \quad w_{3}^{*} w_{2}+z_{2}^{*} z_{3}=-W_{2, \uparrow}, \\
& w_{4}^{*} w_{2}+z_{2}^{*} z_{4}=-W_{1, \downarrow}, \quad w_{4}^{*} w_{1}+z_{1}^{*} z_{4}=W_{1, \uparrow}, \\
& w_{3}^{*} w_{4}+z_{4}^{*} z_{3}=F, \quad w_{1}^{*} z_{2}+w_{2}^{*} z_{1}+w_{3}^{*} z_{4}+w_{4}^{*} z_{3}=0,
\end{aligned}
$$

where $N_{\Lambda}$ represents the number of lattice sites, and $\hat{N}$ is the total particle number operator. Since the total number of particles is a constant of motion, via Eq.(4) we matched the starting Hamiltonian into an unique positive semidefinite form using the conditions presented in Eq.(5).

\section{THE EXACT GROUND STATE AT HALF FILLING}

Let us consider the bond operator

$$
\hat{B}_{\mathbf{i}}^{\dagger}=\alpha_{\uparrow} \hat{c}_{\mathbf{i}, \uparrow}^{\dagger}+\alpha_{\downarrow} \hat{c}_{\mathbf{i}, \downarrow}^{\dagger}+\beta_{\uparrow} \hat{c}_{\mathbf{i}+1, \uparrow}^{\dagger}+\beta_{\downarrow} \hat{c}_{\mathbf{i}+1, \downarrow}^{\dagger}
$$


where $\alpha_{\sigma}$ and $\beta_{\sigma}$ are numerical prefactors. One observes that $\left\{\hat{B}_{\mathbf{i}}^{\dagger}, \hat{B}_{\mathbf{j}}^{\dagger}\right\}=0$ holds for all $\mathbf{i}, \mathbf{j}$ and all $\alpha_{\sigma}, \beta_{\sigma}$.

Let us analyze the wave function

$$
\left|\Psi_{g}\right\rangle=\prod_{\mathbf{i}=1}^{N_{\Lambda}} \hat{B}_{\mathbf{i}}^{\dagger}|0\rangle,
$$

where $|0\rangle$ is the bare vacuum with no fermions present. Since in Eq.(7) one introduces $N_{\Lambda}$ electrons into the system, $\left|\Psi_{g}\right\rangle$ is defined at half filling.

If for all $\mathbf{i}$ one has

$$
\hat{A}_{\mathbf{i}}\left|\Psi_{g}\right\rangle=0
$$

then clearly, $\left|\Psi_{g}\right\rangle$ represents the ground state wave function of $\hat{H}$. Since the fermionic operators from $\hat{A}_{\mathbf{i}}$ in Eq.(3) are present in $\prod_{\mathbf{j}} \hat{B}_{\mathbf{j}}^{\dagger}$ only in $\hat{B}_{\mathbf{i}}^{\dagger}, \hat{B}_{\mathbf{i} \pm 1}^{\dagger}$, it results that in order to satisfy Eq.(8) one must has for all $\mathbf{i}$ sites

$$
\hat{A}_{\mathbf{i}} \hat{B}_{\mathbf{i}}^{\dagger}\left[\hat{B}_{\mathbf{i}-1}^{\dagger} \hat{B}_{\mathbf{i}+1}^{\dagger}\right]|0\rangle=0
$$

from where one finds

$$
\begin{aligned}
& z_{1} \beta_{\downarrow}-z_{3} \alpha_{\downarrow}=0, \quad z_{2} \beta_{\uparrow}+z_{3} \alpha_{\uparrow}=0, \quad z_{1} \alpha_{\uparrow}-z_{4} \beta_{\uparrow}=0, \quad z_{2} \alpha_{\downarrow}+z_{4} \beta_{\downarrow}=0, \\
& w_{1}\left(\alpha_{\uparrow}^{2} \beta_{\downarrow}-\alpha_{\uparrow} \alpha_{\downarrow} \beta_{\uparrow}\right)+w_{3}\left(\beta_{\uparrow}^{2} \alpha_{\downarrow}-\beta_{\uparrow} \beta_{\downarrow} \alpha_{\uparrow}\right)=0, \\
& w_{2}\left(\alpha_{\downarrow}^{2} \beta_{\uparrow}-\alpha_{\uparrow} \alpha_{\downarrow} \beta_{\downarrow}\right)-w_{3}\left(\beta_{\downarrow}^{2} \alpha_{\uparrow}-\beta_{\uparrow} \beta_{\downarrow} \alpha_{\downarrow}\right)=0, \\
& w_{4}\left(\alpha_{\downarrow}^{2} \beta_{\uparrow}-\alpha_{\uparrow} \alpha_{\downarrow} \beta_{\downarrow}\right)+w_{1}\left(\beta_{\downarrow}^{2} \alpha_{\uparrow}-\beta_{\uparrow} \beta_{\downarrow} \alpha_{\downarrow}\right)=0, \\
& -w_{4}\left(\alpha_{\uparrow}^{2} \beta_{\downarrow}-\alpha_{\uparrow} \alpha_{\downarrow} \beta_{\uparrow}\right)+w_{2}\left(\beta_{\uparrow}^{2} \alpha_{\downarrow}-\beta_{\uparrow} \beta_{\downarrow} \alpha_{\uparrow}\right)=0, \\
& -w_{4}\left(\beta_{\downarrow}^{2} \alpha_{\uparrow}-\beta_{\uparrow} \beta_{\downarrow} \alpha_{\downarrow}\right)-w_{1} \beta_{\downarrow}^{2} \beta_{\uparrow}-w_{2} \beta_{\downarrow}^{2} \beta_{\uparrow}=0, \\
& w_{4}\left(\beta_{\uparrow}^{2} \alpha_{\downarrow}-\beta_{\uparrow} \beta_{\downarrow} \alpha_{\uparrow}\right)-w_{1} \beta_{\uparrow}^{2} \beta_{\downarrow}-w_{2} \beta_{\uparrow}^{2} \beta_{\downarrow}=0, \\
& w_{3}\left(\alpha_{\downarrow}^{2} \beta_{\uparrow}-\alpha_{\uparrow} \alpha_{\downarrow} \beta_{\downarrow}\right)-w_{1} \alpha_{\downarrow}^{2} \alpha_{\uparrow}-w_{2} \alpha_{\downarrow}^{2} \alpha_{\uparrow}=0, \\
& -w_{3}\left(\alpha_{\uparrow}^{2} \beta_{\downarrow}-\alpha_{\uparrow} \alpha_{\downarrow} \beta_{\uparrow}\right)-w_{1} \alpha_{\uparrow}^{2} \alpha_{\downarrow}-w_{2} \alpha_{\uparrow}^{2} \alpha_{\downarrow}=0 .
\end{aligned}
$$

In Eq.(10) the first row comes from the coefficients of the terms containing three creation operators created by the product $\hat{A}_{\mathbf{i}} \hat{B}_{\mathbf{i}}^{\dagger}$, while the remaining equations are provided by the rest of the terms from Eq.(9)).

Based on Eqs.(48), the ground state energy is given by $E_{g}=-a_{0}-a_{1} N$. 


\section{THE DEDUCED SOLUTIONS}

In the following one presents the obtained solutions for the system of equations Eqs.(15,10]) denoted by Solutions I. and II. which describe two different regions of the phase diagram.

\section{A. Solution I.}

Taking into consideration $W_{1, \sigma}=W_{2, \sigma}=W$, the parameters of the $\hat{A}_{\mathbf{i}}, \hat{B}_{\mathbf{i}}$ operators become

$$
\begin{aligned}
& z_{2}=-z_{1}, \quad z_{3}=q z_{1} e^{i \alpha}, \quad z_{4}=\frac{z_{1}}{q} e^{-i \alpha}, \quad w_{1}=\frac{z_{1}}{2}\left(\frac{|q|^{2}-1}{q}\right), \\
& w_{2}=-w_{1}, \quad w_{3}=-\frac{z_{1} q^{*}}{q} e^{-i \alpha}, \quad w_{4}=z_{1} e^{i \alpha}, \\
& \alpha_{\uparrow}=\lambda \alpha_{\downarrow}, \quad \beta_{\uparrow}=\lambda q \alpha_{\downarrow} e^{i \alpha}, \quad \beta_{\downarrow}=q \alpha_{\downarrow} e^{i \alpha},
\end{aligned}
$$

where $z_{1}, \alpha, \alpha_{\downarrow}, \lambda, q$ remain arbitrary coefficients at the level of (11). The Hamiltonian parameters turn to have the property $t=t_{\sigma}, V_{1}=V_{\sigma, \sigma}, V_{2}=V_{\sigma,-\sigma}, a_{0}=U N_{\Lambda}, a_{1}=-U$, and one has

$$
W=-\frac{t}{2}, \quad V_{1}=-\frac{J_{z}}{4}, \quad V_{2}=\frac{U+J_{z}}{4}, \quad J_{\perp}=-\frac{U}{2},
$$

where $U>0, t$ and $J_{z}$ can be arbitrary chosen, and the parameters $\left|z_{1}\right|,|q|, \alpha$ becomes

determined by $\left|z_{1}\right|=|t| / \sqrt{U},\left(1+|q|^{2}\right) /|q|=|t| /\left|z_{1}\right|^{2}$, and $e^{-i \alpha}=(q /|q|) \operatorname{sign}(t)$. The coefficients $\lambda$ and $\alpha_{\downarrow}$ remain arbitrary, and the operator constructing the ground state wave function from (7) becomes

$$
\hat{B}_{\mathbf{i}}^{\dagger}=\alpha_{\downarrow}\left[\left(\hat{c}_{\mathbf{i}, \downarrow}^{\dagger}+\lambda \hat{c}_{\mathbf{i}, \uparrow}^{\dagger}\right)+q e^{i \alpha}\left(\hat{c}_{\mathbf{i}+1, \downarrow}^{\dagger}+\lambda \hat{c}_{\mathbf{i}+1, \uparrow}^{\dagger}\right)\right]
$$

\section{B. Solution II.}

If one considers $W_{1, \sigma}=W_{1}, W_{2, \sigma}=W_{2}$ and takes $\nu=z_{3} / w_{4}=-w_{3}^{*} / z_{4}^{*}$ non-zero and real, the parameters of $\hat{A}_{\mathbf{i}}, \hat{B}_{\mathbf{i}}$ operators become

$$
\begin{aligned}
& z_{2}=-z_{1}, \quad z_{3}=P e^{i \alpha} z_{1}, \quad z_{4}=\frac{e^{-i \alpha}}{P} z_{1}, \quad w_{1}=-z_{1}, \\
& w_{2}=z_{1}, \quad w_{3}=-\nu \frac{e^{-i \alpha}}{P} z_{1}, \quad w_{4}=\frac{1}{\nu} P e^{i \alpha} z_{1}, \\
& \alpha_{\uparrow}=\lambda \alpha_{\downarrow}, \quad \beta_{\uparrow}=\lambda P e^{i \alpha} \alpha_{\downarrow}, \quad \beta_{\downarrow}=P e^{i \alpha} \alpha_{\downarrow},
\end{aligned}
$$


where $P=\sqrt{|\nu| /(\sqrt{2}+\operatorname{sign}(\nu))}, \alpha$ is given by $\operatorname{sign}(t)=-e^{-i \alpha}$, and $z_{1}, \alpha_{\downarrow}, \lambda, \nu$ are arbitrary non-zero coefficients. Furthermore one has $a_{1}=a_{0} / N_{\Lambda}=\sqrt{W_{1} W_{2} / 2}\left(2+P^{2}+P^{-2}\right)$, and the Hamiltonian parameters become

$$
\begin{aligned}
& W_{1}=-\operatorname{sign}(t) \frac{\sqrt{2}\left|z_{1}\right|^{2}}{|\nu|} \sqrt{P}, \quad W_{2}=-\operatorname{sign}(t) \sqrt{2}\left|z_{1}\right|^{2}|\nu| \sqrt{P^{-1}}, \\
& J_{\perp}=-\sqrt{2 W_{1} W_{2}}, \quad V_{1}=-\frac{J_{z}}{4}, \quad V_{2}=\frac{J_{z}}{4}-J_{\perp} \\
& t=-\frac{1}{\sqrt{2}}\left(\frac{W_{2}}{|\nu|}+|\nu| W_{1}\right), \quad U=\frac{1+|\nu|^{2}}{|\nu| \sqrt{2 W_{1} W_{2}}}\left(|\nu| W_{1}^{2}+\frac{W_{2}^{2}}{|\nu|}\right),
\end{aligned}
$$

where $\nu, J_{z}$ remain arbitrary, $\operatorname{sign}\left(W_{1}\right)=\operatorname{sign}\left(W_{2}\right)$ must hold, and one has $\left|z_{1}\right|^{2}=$ $\sqrt{W_{1} W_{2} / 2}$. The operator present in the expression of the ground state wave function (17) is given by

$$
\hat{B}_{\mathbf{i}}^{\dagger}=\alpha_{\downarrow}\left[\left(\lambda \hat{c}_{\mathbf{i}, \uparrow}^{\dagger}+\hat{c}_{\mathbf{i}, \downarrow}^{\dagger}\right)-P \operatorname{sign}(t)\left(\lambda \hat{c}_{\mathbf{i}+1, \uparrow}^{\dagger}+\hat{c}_{\mathbf{i}+1, \downarrow}^{\dagger}\right)\right]
$$

so its form is similar to that given in Eq.(13).

\section{Physical properties of the deduced solutions}

For the ground state (7), in both obtained cases (13,16) describing different regions of the phase diagram, the $\hat{B}_{\mathbf{i}}^{\dagger}$ operator is constructed from $\hat{I}_{\mathbf{i}}^{\dagger}=\left(\lambda \hat{c}_{\mathbf{j}, \uparrow}^{\dagger}+\hat{c}_{\mathbf{j}, \downarrow}^{\dagger}\right)$ type of blocks, for which $\hat{I}_{\mathbf{j}}^{\dagger} \hat{I}_{\mathbf{j}}^{\dagger}=0$ holds for arbitrary $\lambda$. Consequently, apart from a normalization constant, $\prod_{\mathbf{i}=1}^{N_{\Lambda}} \hat{B}_{\mathbf{i}}^{\dagger}|0\rangle$ from (7) becomes $\prod_{\mathbf{i}=1}^{N_{\Lambda}} \hat{I}_{\mathbf{i}}^{\dagger}|0\rangle$, hence the normalized ground state in both cases of Solutions I. and II. can be written as

$$
\left|\Psi_{g}\right\rangle=\left(1+|\lambda|^{2}\right)^{-N_{\Lambda} / 2} \prod_{\mathbf{i}=1}^{N_{\Lambda}}\left(\lambda \hat{c}_{\mathbf{j}, \uparrow}^{\dagger}+\hat{c}_{\mathbf{j}, \downarrow}^{\dagger}\right)|0\rangle,
$$

where $\lambda$ is arbitrary, and $N=N_{\Lambda}$ in (17) fixes the filling at half. Denoting by $\langle\ldots\rangle=$ $\left\langle\Psi_{g}|\ldots| \Psi_{g}\right\rangle$ ground state expectation values, and using as usual $\hat{S}_{\mathbf{i}}^{z}=1 / 2\left(\hat{n}_{\mathbf{i}, \uparrow}-\hat{n}_{\mathbf{i}, \downarrow}\right), \hat{S}_{\mathbf{i}}^{+}=$ $\left(\hat{S}^{-}\right)_{\mathbf{i}}^{\dagger}=\hat{c}_{\mathbf{i}, \uparrow}^{\dagger} \hat{c}_{\mathbf{i}, \downarrow}, \hat{S}_{\mathbf{i}}^{ \pm}=\hat{S}_{\mathbf{i}}^{x} \pm i \hat{S}_{\mathbf{i}}^{y}$, and $\hat{\vec{S}}=\sum_{\mathbf{i}} \hat{\vec{S}}_{\mathbf{i}}$ for the spin operators, one finds $\left\langle\hat{S}^{2}\right\rangle=$ $\left(N_{\Lambda} / 2\right)\left(N_{\Lambda} / 2+1\right)$. This result is motivated by the fact that since $\lambda$ is the same on each $\mathbf{i}$, the local spin orientations are the same on each site. Hence the ground state represents a fully saturated ferromagnetic state. Furthermore, since only local Hamiltonian terms contribute in the ground state energy, and independent on $\mathbf{i},\left\langle\hat{n}_{\mathbf{i}, \uparrow}\right\rangle=1-\left\langle\hat{n}_{\mathbf{i}, \downarrow}\right\rangle=|\lambda|^{2} /\left(1+|\lambda|^{2}\right)$, $\left\langle\hat{n}_{\mathbf{i}, \uparrow} \hat{n}_{\mathbf{i}, \downarrow}\right\rangle=0$, the ground state is localized ${ }^{4}$. Consequently, the obtained ground state at 
half filling of the studied one (dispersive) band model is a localized and fully polarized ferromagnet.

Note the important aspect that $\left|\Psi_{g}\right\rangle$ is the ground state only of the full Hamiltonian. Hence not a specific band structure produces here the ferromagnetic behaviour (as for example in the case of the flat band ferromagnetism 23 , or Lieb's degenerate middle band magnetism in a bipartite lattice ${ }^{24}$ ), nor the interactions select the ground state from the minimum energy eigenstates of the kinetic part 25,26 . In the described case the ferromagnetism at half filling can appear in exact terms in an arbitrary dispersive band being only the ground state of the full Hamiltonian, e.g. is provided by an incontestable correlation effect.

As described previously in details $\stackrel{4}{6}$, for a non-integrable model as that studied here, exact ground states can be obtained only on different cuts of the phase diagram corresponding to different decompositions in positive semidefinite form of the starting Hamiltonian. Since for the presented two solutions in Sections V.A, V.B, the expression of the operator $\hat{A}_{\mathbf{i}}$ is different, these solutions correspond to different decompositions, hence provide the deduced ground state in different regions of the phase diagram. The fact that the same solution appears in different regions underlines the stability of the ferromagnetic phase, since finite $\hat{H}$ parameter modifications lead back to the same ordered phase. However, the ferromagnetic localized phase has a finite extension. This is seen from the fact that since $\left|\Psi_{g}\right\rangle$ is not an eigenstate of the non-interacting Hamiltonian, turning off the interactions one recovers the conducting behaviour of a non-interacting dispersive band, e.g. a paramagnetic metal to ferromagnetic insulator transition must be present in the phase diagram of the system.

We further note that resembling ferromagnetic states have been found in similar systems by other methods as well $27,28,29$.

\section{SUMMARY AND CONCLUSIONS}

Single band extended Hubbard models containing spin-spin interactions are studied by a positive semidefinite operator technique, leading to ferromagnetic and localized exact ground states in different regions of the phase diagram via quadratic operators used in the positive semidefinite decomposition leading to an unique operator term in $\hat{H}$. Hence the deduced ground states are minimum energy eigenstate only of the full Hamiltonian, e.g. 
not are connected to a specific band structure, nor are separately eigenstates of the kinetic and interacting parts. The ferromagnetism emerges at half filling in an arbitrary dispersive band, being provided by clear correlation effects.

\section{Acknowledgments}

We kindly acknowledge financial support of the grant OTKA-T48782 of Hungarian Scientific Research Fund, and in case of Zs.G. also from Alexander von Humboldt Foundation.

1 A. Tanaka and H. Tasaki, Phys. Rev. Lett. 98, 116402 (2007).

2 L. G. Sarasua Phys. Rev. B75, 054504 (2007).

3 Z. Gulacsi, A. Kampf and D. Vollhardt, Phys. Rev. Lett. 99, 026404 (2007).

4 Z. Gulacsi and D. Vollhardt, Phys. Rev. Lett. 91, 186401 (2003); and Phys. Rev. B72, 075130 (2005).

5 L. G. Sarasua and M. A. Continentino, Phys. Rev. B69, 073103 (2004).

6 Z. Gulacsi and M. Gulacsi, Phys. Rev. B 73, 014524 (2006).

7 Z. Gulacsi, Phys. Rev. B69, 054204 (2004).

8 Z. Gulacsi and M. Gulacsi, Phys. Rev. Lett. 73, 3239 (1994).

9 E. Kovacs and Z. Gulacsi, Jour. of Phys. A 38, 10273 (2005); Phil. Mag. 86, 1997 (2006); Phil. Mag. 86, 2073 (2006).

10 U. Brandt and A. Giesekus, Phys. Rev. Lett. 68, 2648 (1992); R. Strack and D. Vollhardt, Phys. Rev. Lett. 70, 2637 (1993).

11 E. Kovacs and Z. Gulacsi, Phil. Mag. B81, 341 (2001).

12 R. Chan and M. Gulacsi, Phil. Mag. 84, 1265 (2004); Phil. Mag. Lett. 81, 673 (2001); Jour. of Superconductivity 14, 651 (2001).

13 A. Klumper, A Schadschneider and J. Zittartz. Europhys. Lett. 24, 293 (1993).

14 Z. Gulacsi, Phys. Rev. B66, 165109 (2002).

15 R. Strack and D. Vollhardt, Phys. Rev. Lett. 72, 3425 (1994).

16 Z. Gulacsi, Phil. Mag. Lett. 84, 405 (2004).

17 M. Gulacsi, Advances in Physics 53, 769 (2004); Phil. Mag. 86, 1907 (2006). 
18 E. Dagotto, Rev. Mod. Phys. 66, 763 (1994).

19 J. de Boer, V. E. Korepin and A. Schadschneider, Phys. Rev. Lett. 74, 789 (1995).

20 M. Kollar, R. Strack and D. Vollhardt, Phys. Rev. 53, 9225 (1996).

21 D. K. Campbell, J. T. Gammel, and E. Y. Loh Jr., Phys. Rev. B42, 475 (1990).

22 S. V. Vonsovsky and M. I. Katsnelson, Jour. of Phys. C12, 2043 and 2055 (1979).

23 A. Mielke, Jour. of Phys. A24, L73 (1991); H. Tasaki, Phys. Rev. Lett. 69, 1608 (1992).

24 E. H. Lieb, Phys. Rev. Lett. 62, 1201 (1989).

25 We include here also bond-charge terms in the kinetic part of $\hat{H}$.

26 This is the case when several positive semidefinite operators are used in the expression of the transformed Hamiltonian.

27 A. Juozapavicius et al., Phil. Mag. B82, 1211 (2002).

28 I. P. McCulloch et al. Phys. Rev. B65, 052410 (2002) and Phil. Mag. Lett. 81, 869 (2001).

29 G. Honner and M. Gulacsi, Phys. Rev. B58, 2662 (1998). 